\title{
Forsythoside A Alleviates High Glucose-Induced Oxidative Stress and Inflammation in Podocytes by Inactivating MAPK Signaling via MMPI2 Inhibition
}

\author{
Xiaohong Quan ${ }^{1, *}$ \\ Huihui Liu',* \\ Dongmei $\mathrm{Ye}^{2}$ \\ Xinling Ding ${ }^{3}$ \\ Xiulan $\mathrm{Su}^{4}$
}

'Experiment \& Teaching Center for Basic Medicine, Chifeng University School of Basic Medical Sciences, Chifeng, Inner Mongolia, 024000, People's Republic of China; ${ }^{2}$ Core Facility Center for Functional Experiments, CUSBMS, Chifeng University School of Basic Medical Sciences, Chifeng, Inner Mongolia, 024000, People's Republic of China; ${ }^{3}$ Department of Human Anatomy, CUSBMS, Chifeng University School of Basic Medical Sciences, Chifeng, Inner Mongolia, 024000, People's Republic of China; ${ }^{4}$ Clinical Research Center for Medical Sciences, IMMU, Chifeng University School of Basic Medical Sciences, Chifeng, Inner Mongolia, 024000, People's Republic of China

*These authors contributed equally to this work

Correspondence: Xiaohong Quan Experiment \& Teaching Center for Basic Medicine, Chifeng University School of Basic Medical Sciences, No. I Yingbin Road, Chifeng City, Inner Mongolia, 024000, People's Republic of China Email quanxiaohongxh@I63.com
Background: Podocyte injury serves an important role during the progression of diabetic nephropathy (DN). The aim of this study was to investigate the effects of forsythoside A (FA) on high glucose (HG)-induced podocyte injury and to identify the possible mechanisms.

Methods: MPC-5 podocytes were cultured under HG conditions. After exposure to different doses of FA, cell viability and apoptosis were respectively evaluated with CCK-8 assay and flow cytometry. Then, the levels of oxidative stress-related markers and inflammatory factors were examined by corresponding kits. Western blot analysis was employed to detect the expression of Nox2, Nox4, COX-2, iNOS and matrix metalloproteinases 12 (MMP12). Subsequently, MMP12 was overexpressed to assess whether the effects of FA on HGstimulated podocyte injury were mediated by MMP12 and MAPK signaling.

Results: Results indicated that FA dose-dependently elevated cell viability, reduced cell apoptosis in HG-induced MPC-5 cells. Additionally, FA significantly inhibited oxidative stress, which could be certified by decreased content of malondialdehyde (MDA), enhanced activities of superoxide dismutase (SOD) and catalase (CAT), and downregulated expression of Nox 2 and Nox4. Moreover, notably reduced levels of tumor necrosis factor (TNF)- $\alpha$, interleukin (IL)-1 $\beta$ and IL- 6 were observed in FA-treated MPC-5 cells under HG conditions, accompanied by decreased COX-2 and iNOS expression. Remarkably, FA suppressed MMP12 expression in a dose-dependent manner, and the effects of FA on MPC-5 cells exposed to HG were partially counteracted by MMP12 overexpression. Mechanically, FA inactivated the expression of phospho-ERK (p-ERK), p-p38 and p-JNK, which was restored after MMP12 overexpression.

Conclusion: These findings demonstrate a protective mechanism of FA by inactivating MAPK signaling via MMP12 inhibition in HG-induced podocyte injury, providing a promising therapeutic candidate for the treatment of DN.

Keywords: diabetic nephropathy, high glucose, oxidative stress, inflammation, apoptosis

\section{Introduction}

Diabetic nephropathy (DN), one of the most common diabetic complications, is the principal cause of chronic kidney disease and end-stage renal disease which leads to global health and socioeconomic burdens on human beings. ${ }^{1,2}$ It is estimated that approximately $30-50 \%$ of the patients with type 1 or type 2 diabetes develop 
symptoms of DN during their lifetime, which results in a high mortality rate. ${ }^{3,4}$ The conventional approaches to treating DN are strict control of hyperglycemia and blockage of renin-angiotensin-aldosterone system, which can only cut down but not eliminate DN risk. ${ }^{5}$ Therefore, it is particularly important for us to more comprehensively understand the pathogenesis of DN development in order to find new and effective treatment options for treatment of this disease.

Podocytes are a kind of highly specialized and terminally differentiated tubular epithelial cells, which can constitute glomerular filtration barrier. ${ }^{6,7}$ A considerable body of evidence indicates that the functional and structural injuries of podocytes representing a typical characteristic of DN occur in the early stages of this disease. ${ }^{8,9}$ Further, damaged podocytes cause the impairment of selective glomerular filtration function and prompts the formation of proteinuria. ${ }^{10}$ Existing studies have shown that the generation of reactive oxygen species (ROS), which is induced by persistent hyperglycemia, invariably brings about the impairment of the antioxidant defense systems, oxidative stress and inflammation. ${ }^{11,12}$ Therefore, employing novel therapies that possess antioxidant and antiinflammatory properties may afford protection against DN.

Forsythoside A (FA), one of the main bioactive components isolated from air-dried fruits of Forsythia suspensa, has been reported to possess multiple beneficial properties, such as antioxidant, anti-bacterial, antiinflammatory, antiviral and antipyretic characteristics. ${ }^{13-16}$ It was found that Forsythia suspensa extract, where FA might be the major antioxidant constituent, attenuates diquat-induced oxidative stress. ${ }^{17}$ Compelling evidence indicates that FA could alleviate lipopolysaccharide-induced inflammation via upregulating miR-124 expression. $^{18}$ FA plays antiinflammatory role in staphylococcus aureus-stimulated primary bovine mammary epithelial cells through inactivation of NF-kappa B and MAPKs pathways. ${ }^{19}$ Research has proposed that FA alleviates renal damage in adriamycin-induced nephropathy rats. ${ }^{20}$ Additionally, the SwissTargetPrediction website (http://www.swisstarget prediction.ch/) predicts that FA can be combined to macrophage metalloelastase 12 (MMP12), which is a protein of the matrix metalloproteinase family. ${ }^{21}$ Emerging evidence supports that the levels of plasma MMP12 are closely related to atherosclerotic burden and symptomatic cardiovascular disease in subjects

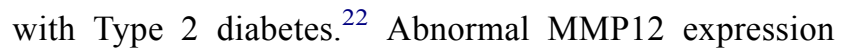

is associated with an increased risk of $\mathrm{DN}^{23}$ Therefore, we focused on whether FA might exert protective effects in high glucose (HG)-induced podocytes by regulating MMP12 and related signaling pathway.

The present study used HG-induced mouse podocytes (MPC-5 cells) model to explore the protective effects of FA on oxidative stress, inflammation and apoptosis and identify the potential mechanisms.

\section{Materials and Methods Cell Culture}

Conditionally immortalized mouse podocytes MPC-5 cell line were obtained from Cell Bank of the Chinese Academy of Sciences (Shanghai, China). Cells were maintained in RPMI-1640 (Sigma, St. Louis, MO, USA) medium supplemented with 10\% FBS (HyClone Laboratories, Logan, UT, USA) and $10 \mathrm{U} / \mathrm{mL}$ of mouse recombinant interferon- $\gamma$ (IFN- $\gamma$ ) (PeproTech, California, USA). The incubator was humidified and maintained at the temperature of $33^{\circ} \mathrm{C}$, with $5 \% \mathrm{CO}_{2}$ and $95 \%$ room air. To induce differentiation, MPC-5 cells were maintained in RPMI 1640 medium without IFN- $\gamma$ at $37^{\circ} \mathrm{C}$ for $10-14$ days.

\section{Treatment}

Prior to stimulation, the differentiated podocytes were serum-starved overnight to induce quiescence. Then, MPC-5 cells were incubated with medium containing normal glucose (NG, $5.5 \mathrm{mM}$ glucose), $5.5 \mathrm{mM}$ glucose plus $22.5 \mathrm{mM}$ mannitol (MA) as the osmolality control, or $\mathrm{HG}$ (25 mM glucose) at $37^{\circ} \mathrm{C}$ for $24 \mathrm{~h}$. Different doses of FA $(2.5,5$ and $10 \mu \mathrm{g} / \mathrm{mL})$ were used to treat cells for 24 $\mathrm{h}$ after glucose challenge. FA (purity of 98.60\%) was purchased from Must Bio-Technology (Chengdu, China).

\section{Cell Transfection}

MPC-5 cells $\left(1 \times 10^{6}\right.$ per well $)$ in logarithmic phase were collected and seeded in 6-well plates. When the cell confluence rate reached $85 \%$, MMP12 overexpression plasmid and the empty vector were transfected into cells by means of lipofectamine $2000^{\circledR}$ reagent (Invitrogen; Thermo Fisher Scientific, Inc.) according to the manufacturer's recommendations. Forty-eight hours after post-transfection, the expression of MMP12 was tested with Western blot assay.

\section{Cell Viability Assay}

A Cell Counting Kit-8 (CCK-8) assay (Beyotime Institute of Biotechnology) was adopted to assess MPC-5 cell 
viability following the product instructions. Briefly, cells were plated into a 96 -well plate $\left(5 \times 10^{3}\right.$ cells per well) and cultured at $37^{\circ} \mathrm{C}$ for $48 \mathrm{~h}$ prior to the addition of $10 \mu \mathrm{L}$ CCK-8 solution. After $2 \mathrm{~h}$ of incubation, cell absorbance at $450 \mathrm{~nm}$ was evaluated using the microplate reader (BioRad Laboratories, Richmond, CA, USA).

Cell death was evaluated by examining the activity of lactate dehydrogenase (LDH) in the culture medium with the LDH assay kit purchased from Nanjing Jiancheng Bioengineering Institute (Nanjing, China) in accordance with the manufacturer's descriptions. Absorbance was read at $450 \mathrm{~nm}$ using a microplate reader (Bio-Rad Laboratories, Richmond, CA, USA).

\section{Terminal Deoxynucleotidyl}

\section{Transferase-Mediated Deoxyuridine} Triphosphate (dUTP)-Biotin Nick End Labeling (TUNEL) Apoptosis Staining

Apoptotic cells were examined by an apoptosis detection kit (Roche, Basel, Switzerland) in accordance with the manufacturer's guidelines. Briefly, cells were stained by a TUNEL kit, followed by the counterstaining with 4', 6-diamidino-2-phenylindole (DAPI) for the nuclei. The staining was observed under a fluorescence microscope (Olympus Corporation, magnification, x200). TUNELpositive cells were captured under an fluorescence microscope (Olympus Corporation, magnification, x200).

\section{Test for Oxidative Stress-Related Markers}

The content of malondialdehyde (MDA) and the activities of superoxide dismutase (SOD) and catalase (CAT) were detected using specific commercial assay kits (Nanjing Jiancheng Bioengineering Institute, Nanjing, China) according to the manufacturer's recommendations. Subsequently, the absorbance was measured by a microplate reader (Bio-Rad Laboratories, Richmond, CA, USA).

\section{Assessment of Inflammatory Factors}

Following indicated transfection and treatment, the supernatants of podocytes were collected. The contents of tumor necrosis factor (TNF)- $\alpha$, interleukin (IL)-1 $\beta$ and IL-6 were evaluated by means of Enzyme-linked immunosorbent (ELISA) kits (Shanghai Xitang Biotechnology Co., Ltd., Shanghai, China) based on the producer's protocols. Absorbance readings of samples were detected at $450 \mathrm{~nm}$ with a microplate reader (Bio-Rad Laboratories, Richmond, CA, USA).

\section{Reverse Transcription-Quantitative Polymerase Chain Reaction (RT-qPCR) Analysis}

Trizol reagent (Invitrogen) was used to extract the total RNA from MPC-5 cells according to the manufacturer's instructions. Then, RNA was reverse transcribed into cDNA using a Reverse Transcription kit (Beijing TransGen Biotech, Beijing, China). qPCR was conducted using SYBR Premix Ex Taq (TaKaRa, Dalian, China) on Applied Biosystems 7500 (Applied Biosystems, Foster City, California). The primer sequences used in this study were as follows: MMP12, forward 5'CATGAAGCGTGAGGATGTAGAC-3', reverse 5'TGGGCTAGTGTACCACCTTTG-3'; GAPDH, forward 5'-AATGGATTTGGACGCATTGGT-3', reverse 5'TTTGCACTGGTACGTGTTGAT-3'. Analysis of gene expression employed relative quantification using $2^{-\Delta \Delta C T}$ method. ${ }^{24}$ GAPDH was used as an internal reference gene.

\section{Western Blot Analysis}

MPC-5 podocytes in each group were lysed by RIPA buffer (Beyotime Institute of Biotechnology) on the ice to extract the total protein. A bicinchoninic acid assay kit (Pierce; Thermo Fisher Scientific, Inc.) was used to determine the concentrations of total proteins. The same amount of protein (50 $\mu \mathrm{g} /$ lane) was subjected to $10 \%$ SDS-PAGE electrophoresis. The separated proteins were then transferred to PVDF membranes. After blocking with 5\% skimmed milk for 1.5 $\mathrm{h}$ at room temperature, these membranes were incubated overnight at $4^{\circ} \mathrm{C}$ with primary antibodies. Following primary incubation, these blots were immersed in HRP-conjugated secondary antibodies (Cell Signaling Technology; cat. no. 7074S). The immunoreactive protein bands were visualized using the enhanced chemiluminescence kit (Amersham Pharmacia Biotech, Amersham, UK). Anti-Nox2 (cat. no. ab129068), anti-Nox4 (cat.no. ab154244) and anti-MMP12 antibodies were the products of Abcam Company (Cambridge, UK). Anti-cyclooxygenase-2 (COX-2; cat. no. 12282T), anti-inducible nitric oxide synthase (iNOS; cat. no. 13120S), anti-phospho-ERK (p-EKR; cat. no. 4370T), antiERK (cat. no. 4695T), anti-p-p38 (cat. no. 4511T), anti-p38 (cat. no. 8690T), anti-p-JNK (cat. no. 9255S), anti-JNK (cat. no. 9258T) and anti-GAPDH (cat. no. 5174T) antibodies were purchased from Cell Signaling Technology (Boston, 
MA, USA). Protein bands were analyzed using ImageJ software (National Institutes of Health). GAPDH was used as the loading control.

\section{Statistical Analysis}

Data are presented as the mean \pm standard deviation. A comparison among multiple groups was performed by a one-way analysis of variance (ANOVA) followed by Tukey's post-hoc test. P-values of $<0.05$ were taken as statistically significant.

\section{Results}

FA Treatment Increases Cell Viability and Decreases Cell Apoptosis in HG-Induced Podocytes

The chemical structural formula of FA was presented in Figure 1A. To study the effects of FA on HG-induced podocytes MPC-5 cell line, firstly, cell viability was examined with CCK-8 assay after cells being treated with different concentrations of FA. As shown in Figure $1 \mathrm{~B}$, there was no significant effect on cell viability in
A

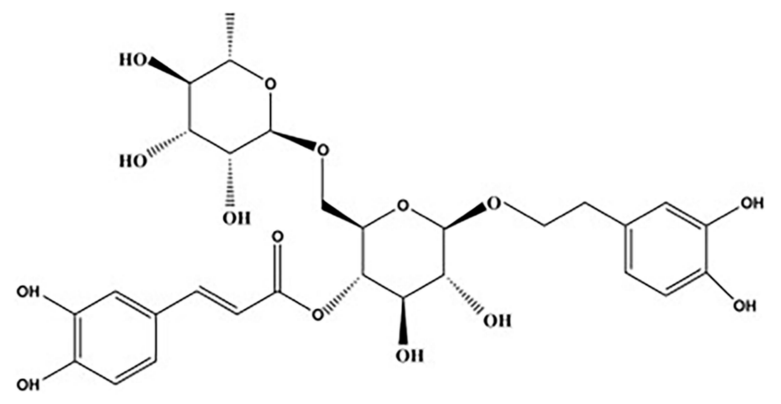

B

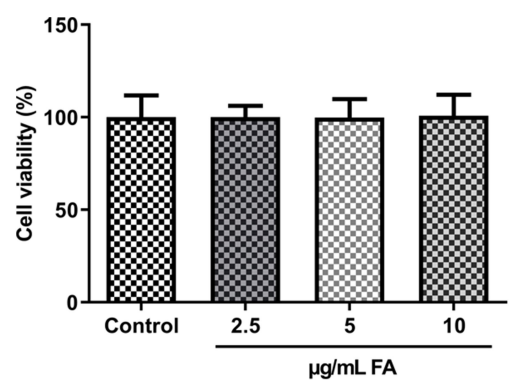

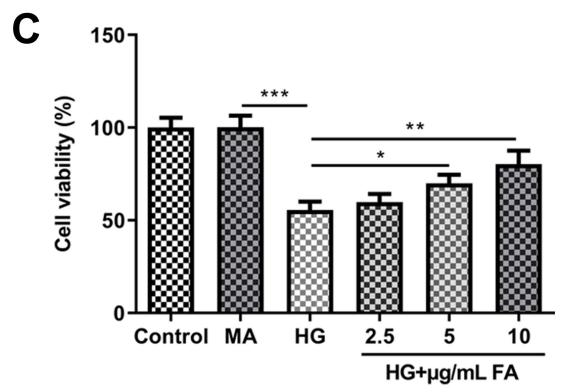
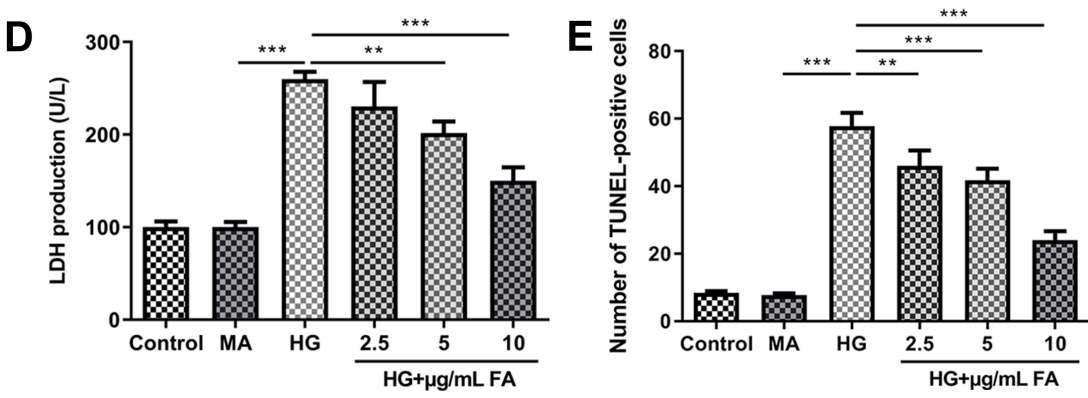

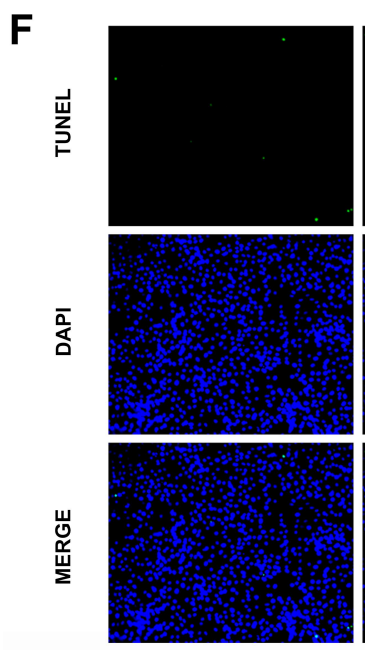

Control
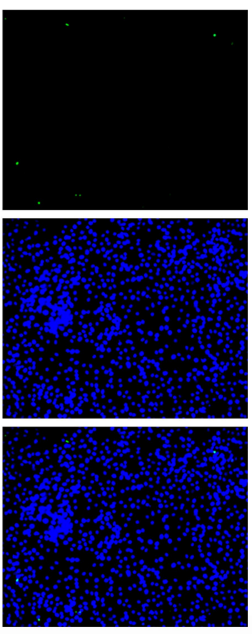

MA
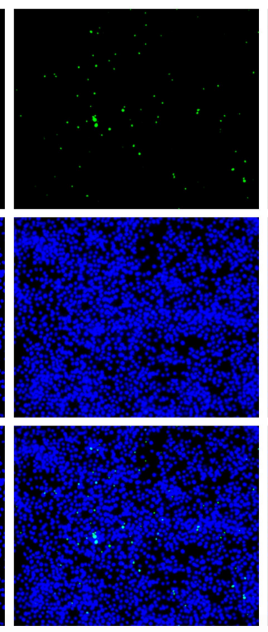

HG
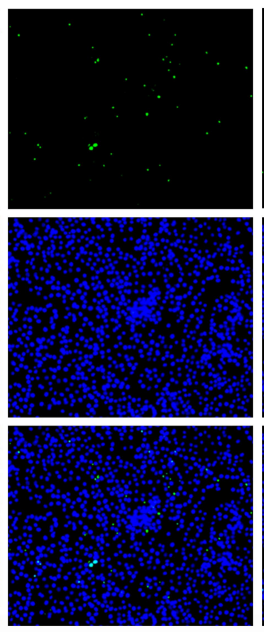

2.5

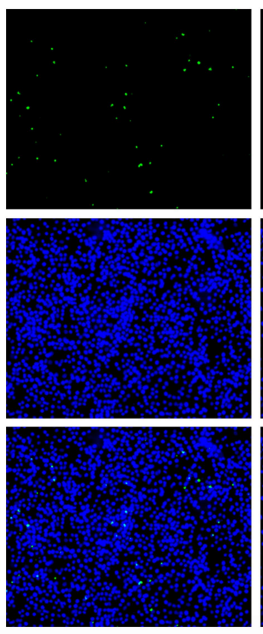

5
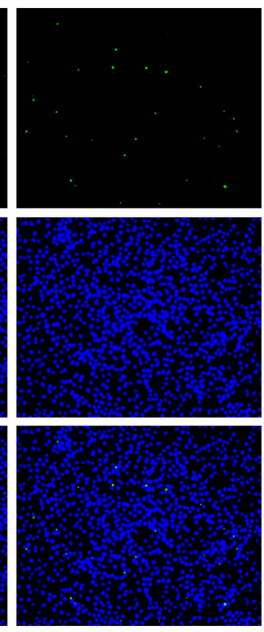

10

$H G+\mu g / m L F A$

Figure I FA treatment intensified cell viability and suppressed cell apoptosis in HG-induced podocytes. (A) The chemical structural formula of FA. (B) Cell viability was tested with CCK-8 assay after treatment with different doses of FA. (C) Cell viability was evaluated using CCK-8 assay in the presence or absence of FA under HG condition. (D) The activity of LDH was determined by means of LDH assay kit. (E and $\mathbf{F}$ ) Cell apoptosis was assessed by TUNEL assay. $* \mathrm{P}<0.05$, $* * \mathrm{P}<0.0 \mathrm{I}$, $* * * \mathrm{P}<0.00 \mathrm{I}$. 
response to FA with $2.5,5$ and $10 \mu \mathrm{g} / \mathrm{mL}$ relative to the control group. Then, it was found that $\mathrm{HG}$ exposure notably decreased cell viability compared with the mannitol control (MA) group (Figure 1C). By contrast, FA dose-dependently elevated cell viability as comparison to the HG induction group. Additionally, the activity of LDH was remarkably intensified when MPC-5 cells were stimulated with medium containing $\mathrm{HG}$ (Figure 1D). However, FA treatment conspicuously reduced LDH activity in a dose-dependent manner relative to the HG group. Subsequently, cell apoptosis was evaluated by means of TUNEL assay. As what is observable from Figure $1 \mathrm{E}$ and $\mathrm{F}, \mathrm{HG}$ exposure significantly enhanced the number of apoptotic cells compared with the MA group, which was reversed by FA intervention. These results indicate that FA treatment elevates cell viability and inhibits cell apoptosis in HG-induced podocytes.

\section{FA Treatment Notably Alleviates Oxidative Stress and Inflammation in Podocytes Under HG Condition}

To explore the effects of FA on oxidative stress in $\mathrm{HG}$ stimulated podocytes, the levels of oxidative stressrelated markers were detected with corresponding kits. As exhibited in Figure $2 \mathrm{~A}-\mathrm{C}, \mathrm{HG}$ challenge conspicuously increased the content of MDA whereas decreased the activities of SOD and CAT antioxidases relative to the MA group, which was restored by FA treatment. Consistently, as compared to the MA group, the expression of NADPH oxidase (Nox)2 and Nox4 was remarkably upregulated in the HG-treated group, whereas FA dose-dependently downregulated the levels of these two proteins relative to the HG group (Figure 2D). Concurrently, it was found that $\mathrm{HG}$ stimulation significantly elevated the concentrations of TNF- $\alpha$, IL$1 \beta$ and IL-6 compared with mannitol control group, and the pro-inflammatory impact of $\mathrm{HG}$ induction in MPC-5 cells was partially counteracted after FA intervention (Figure 2E-G). Additionally, FA treatment dramatically reversed the promoted effect of $\mathrm{HG}$ stimulation on the expression of COX-2 and inducible nitric oxide synthase (iNOS) (Figure 2H). Overall, these data suggest that intervention with FA markedly attenuates HG-induced oxidative stress and inflammation in podocytes.

\section{FA Inactivates MMPI2 Expression in HG-Induced Podocytes}

To investigate the potential mechanisms of FA in DN model in vitro, the expression of MMP12 was tested by Western blotting. It was observed that HG induction significantly upregulated MMP12 expression as compared to the MA group, while FA dose-dependently downregulated the level of MMP12 (Figure 3A and B). Then, MMP12 was overexpressed by transfection with MMP12 plasmid. Results obtained in Figure 3C and D revealed that MMP12 expression level was remarkably elevated after transfection with MMP12 plasmid compared with the empty group. These data provide evidence that FA treatment inactivates MMP12 expression in HG-induced podocytes.

\section{MMPI2-Upregulation Conspicuously Restores the Impact of FA Treatment on HG-Induced Podocyte Injury}

To further clarify the mechanism of FA and MMP12 in MPC-5 cells under HG exposure, cell viability and cell apoptosis were tested firstly. As displayed in Figure 4A and B, MMP12 overexpression notably decreased cell viability and enhanced LDH activity as compared to the empty group. Then, it was found that the gain-function of MMP12 notably promoted cell apoptosis in FA-treated MPC-5 cells under $\mathrm{HG}$ condition when compared with Oe-NC group (Figure 4C and D). Additionally, significantly increased MDA content and decreased SOD and CAT activities were noticed following overexpression of MMP12 (Figure 5AC). Meanwhile, the expression of Nox 2 and Nox 4 presented marked upregulation after MMP12 overexpression (Figure 5D). Moreover, MMP12 overexpression conspicuously promoted the section of inflammatory factors TNF- $\alpha$, IL- $\beta$ and IL-6 relative to the empty control group in MPC-5 cells stimulated by $\mathrm{HG}$ and FA (Figure $5 \mathrm{E}-\mathrm{G}$ ). As expected, notably intensified COX-2 and iNOS expression levels were observed after transfection with MMP12 plasmid (Figure 5H). These observations reveal that FA attenuates HG-induced cell apoptosis, oxidative stress and inflammation by inhibiting MMP12 expression.

\section{FA Alleviates HG-Induced Podocyte Injury by Inactivating MAPK Signaling via MMPI 2 Inhibition}

Finally, the expression of proteins in MAPK signaling was evaluated by means of Western blot analysis. As 

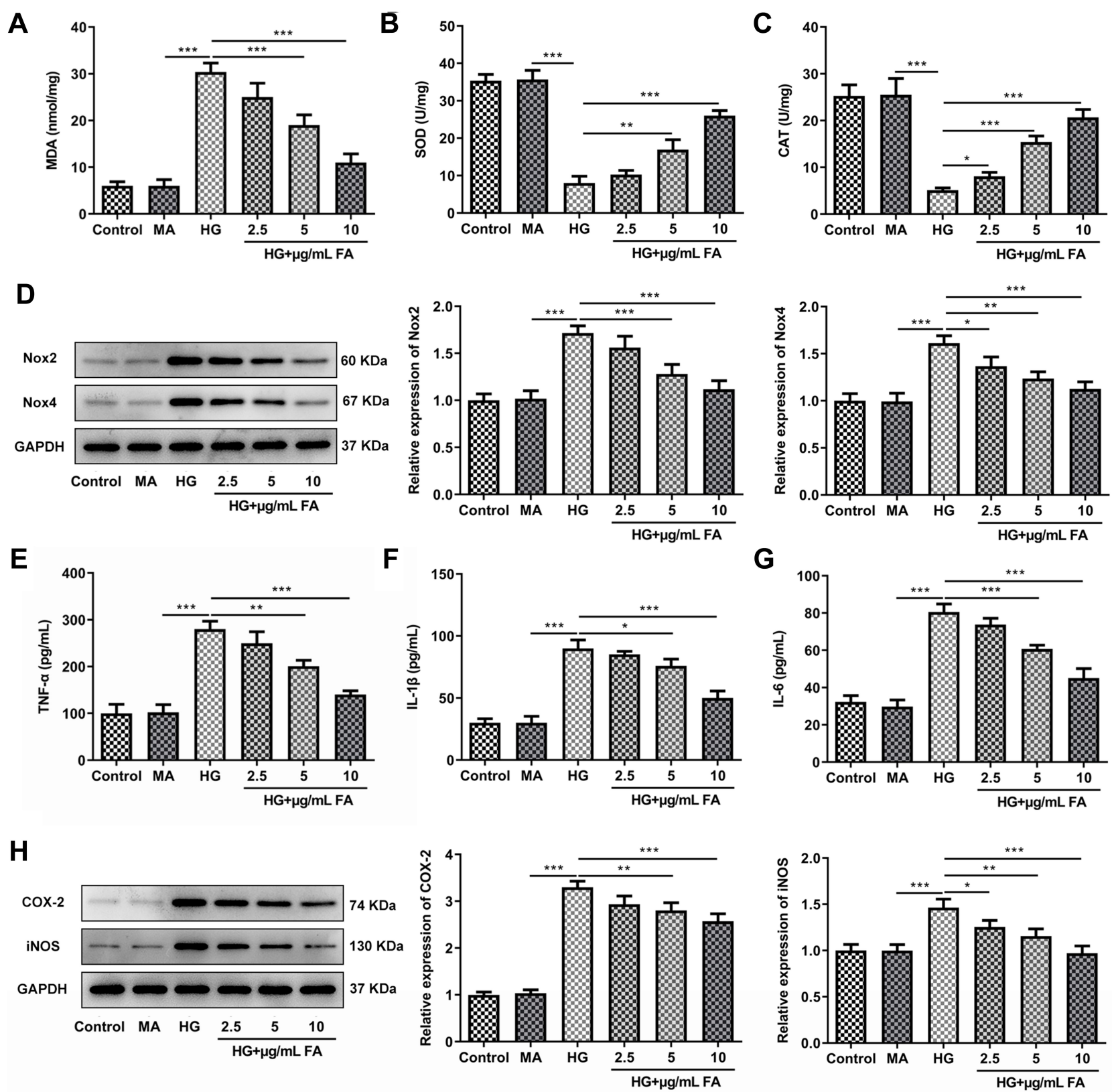

Figure 2 FA treatment significantly alleviates oxidative stress and inflammation in HG-induced MPC-5 cells. (A-C) The levels of MDA, SOD and CAT were tested by means of corresponding commercially available kits. (D) Western blot analysis was employed to examine the expression of Nox2 and Nox4. (E-G) The concentrations of TNF- $\alpha$, IL-I $\beta$ and IL-6 were evaluated with ELISA kits. (H) The expression of COX-2 and iNOS was determined with Western blotting. *P<0.05, **P<0.0I, ***P<0.00I.

shown in Figure 6, HG stimulation notably upregulated the expression of p-ERK, p-p38 and p-JNK as compared to the MA group. However, FA intervention downregulated the levels of above-mentioned proteins in MPC-5 cells exposed to HG. Interestingly, reduced expression of p-ERK, p-p38 and p-JNK after FA treatment was partially restored following MMP12 overexpression. To conclude, these findings suggests that FA relieves HGinduced podocyte injury by inactivating MAPK signaling via MMP12 inhibition.

\section{Discussion}

DN, evoked by persistent hyperglycemia, is a frequent cause of morbidity and death among patients with diabetes. ${ }^{25}$ Podocytes are terminally differentiated and highly specialized cells that play a significant role in maintaining the glomerular structure and filtration barrier. ${ }^{26}$ Due to limited division capacities, podocyte injury and loss is a critical factor contributing to the progression of $\mathrm{DN}^{27}$ Traditional Chinese medicine (TCM) has become increasingly important in the treatment 
A

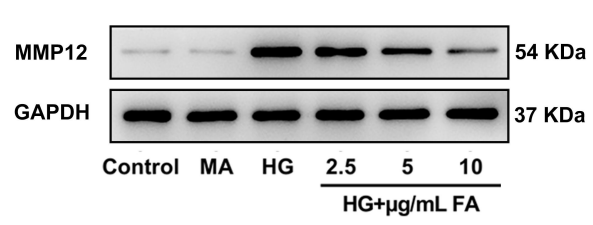

C
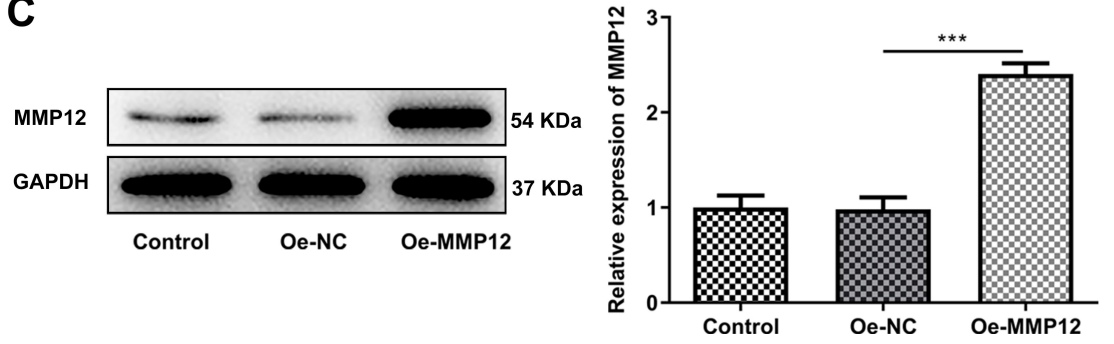

B

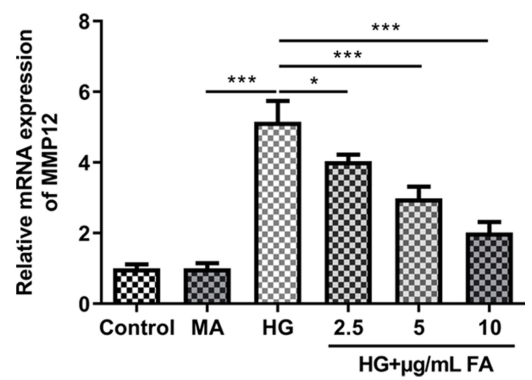

D

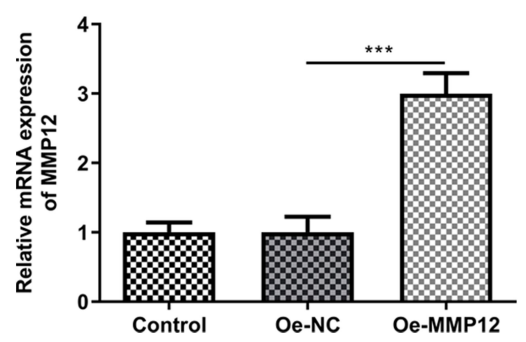

Figure 3 FA reduced MMPI 2 expression level in HG-induced MPC-5 cells. (A and B) The expression of MMPI 2 was respectively evaluated using Western blotting and RTqPCR. (C and D) Western blot assay and RT-qPCR were used to detect MMPI 2 level after transfection. $* P<0.05, * * * P<0.001$.

A

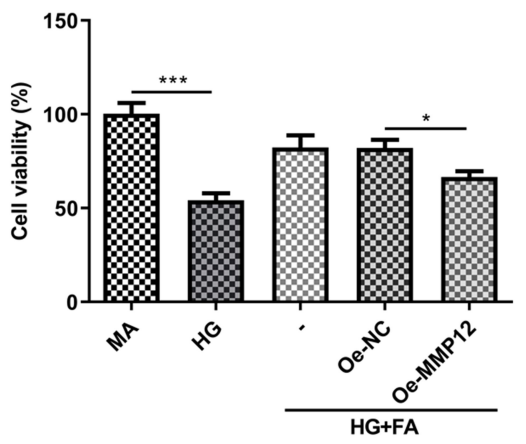

D
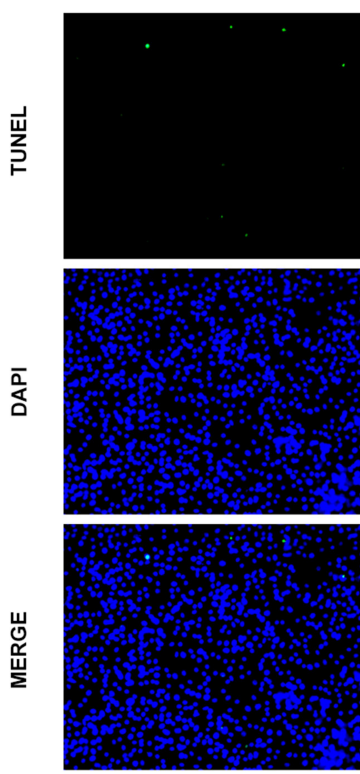

MA
B
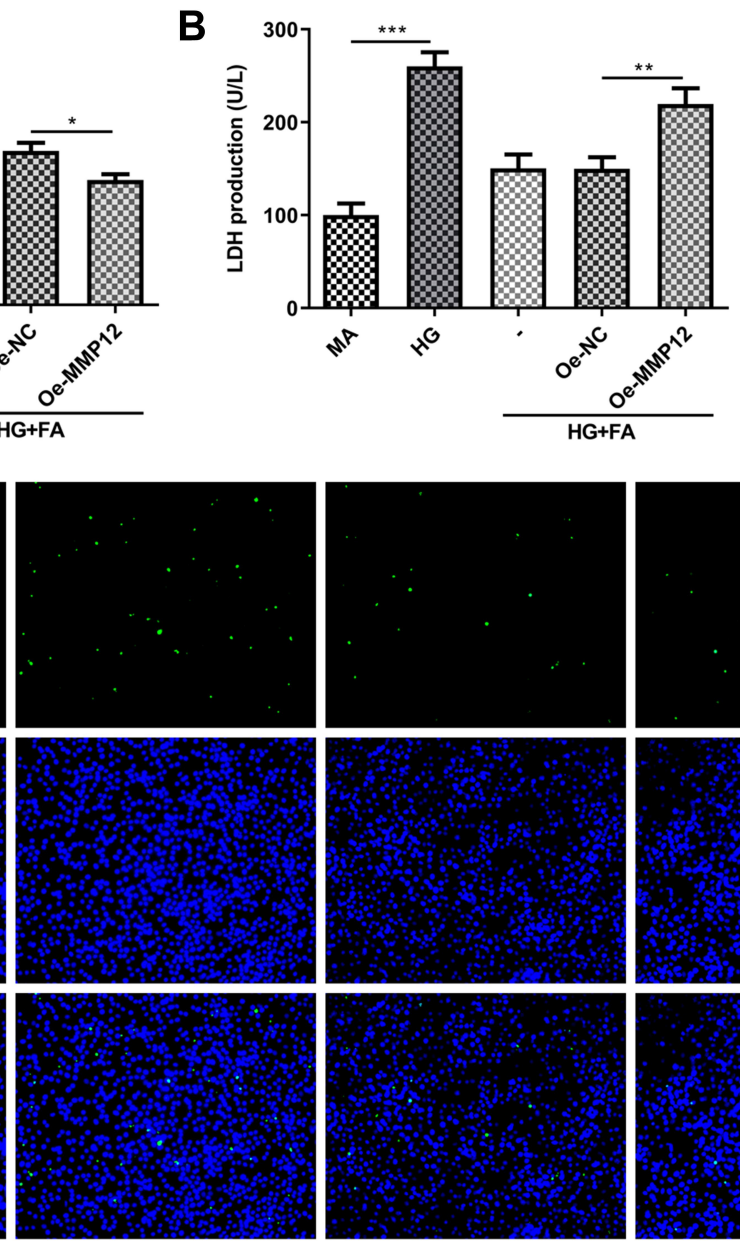

HG
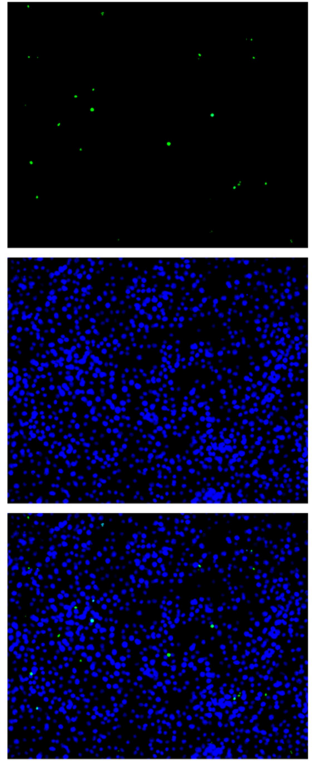

HG+FA
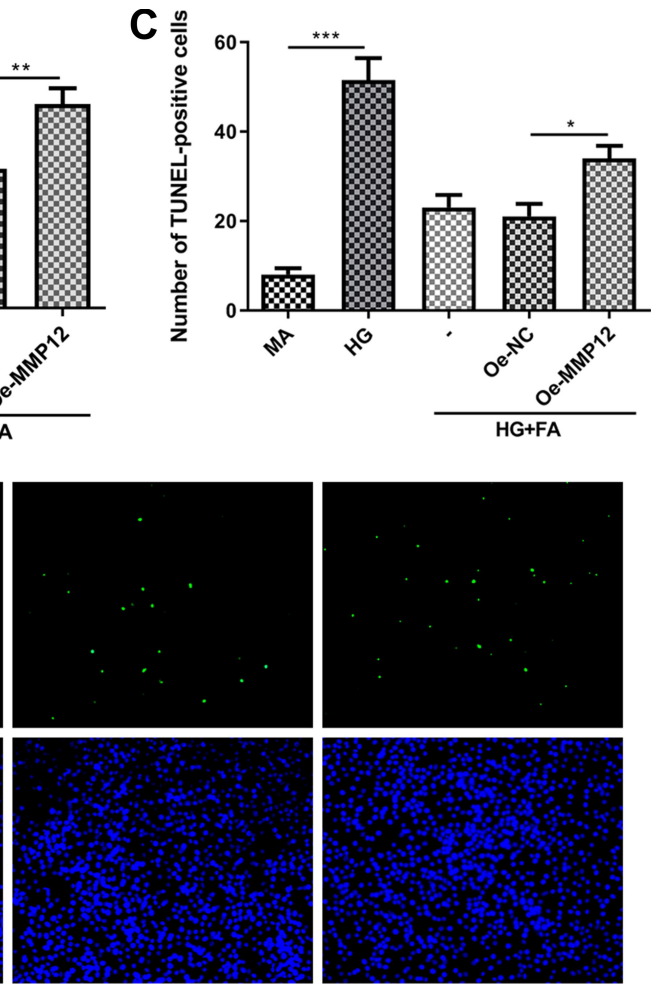

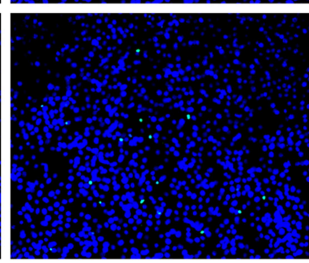

$\mathrm{HG}+\mathrm{FA}+\mathrm{Oe}-\mathrm{NC}$

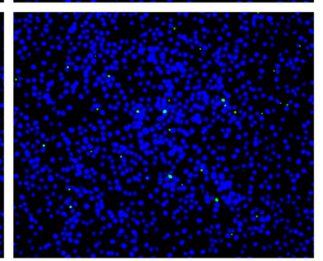

HG+FA+Oe-MMP12

Figure 4 MMPI 2 overexpression partially reversed the impact of FA treatment on cell viability and cell apoptosis in HG-induced podocyte. (A) Cell viability was tested with CCK-8 assay. (B) LDH activity was measured using LDH assay kit. (C and D) TUNEL assay was adopted for evaluation of cell apoptosis. $* \mathrm{P}<0.05$, $* * \mathrm{P}<0.0 \mathrm{I}$, $* * * \mathrm{P}<0.00 \mathrm{I}$. 
A
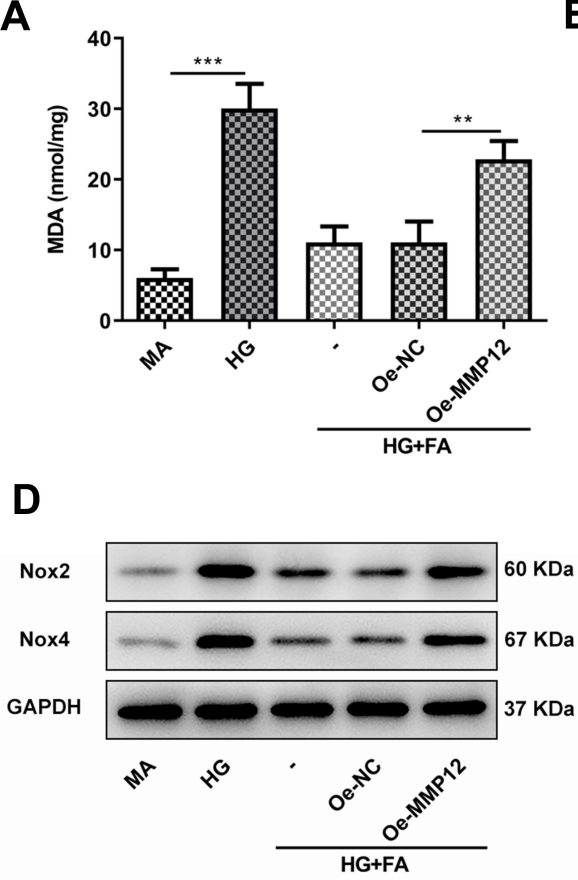

E

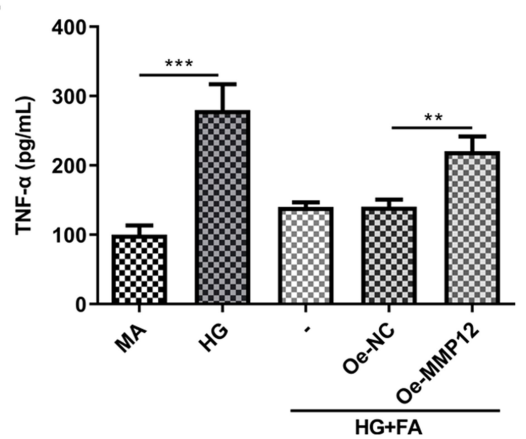

H

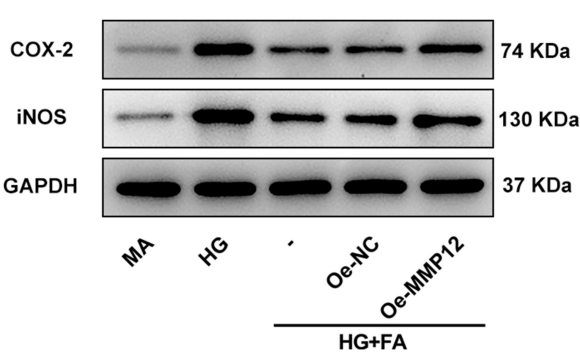

B
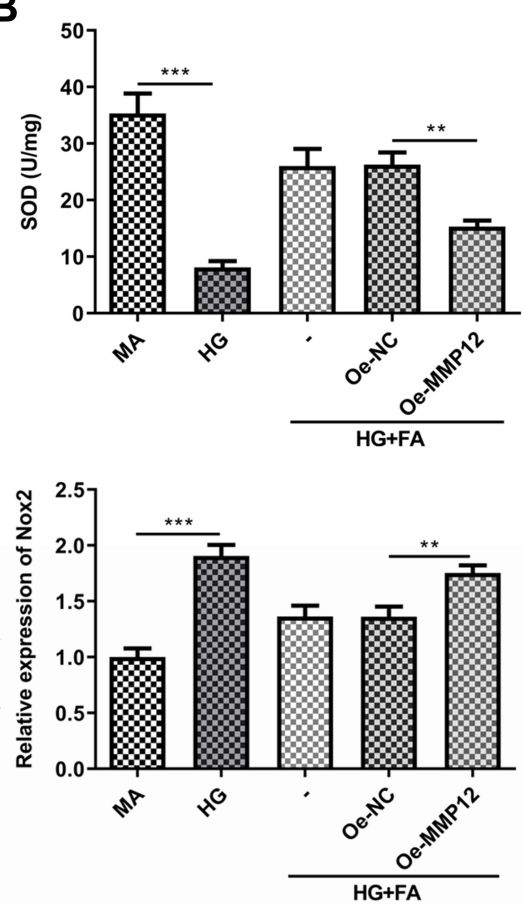

$\mathbf{F}$
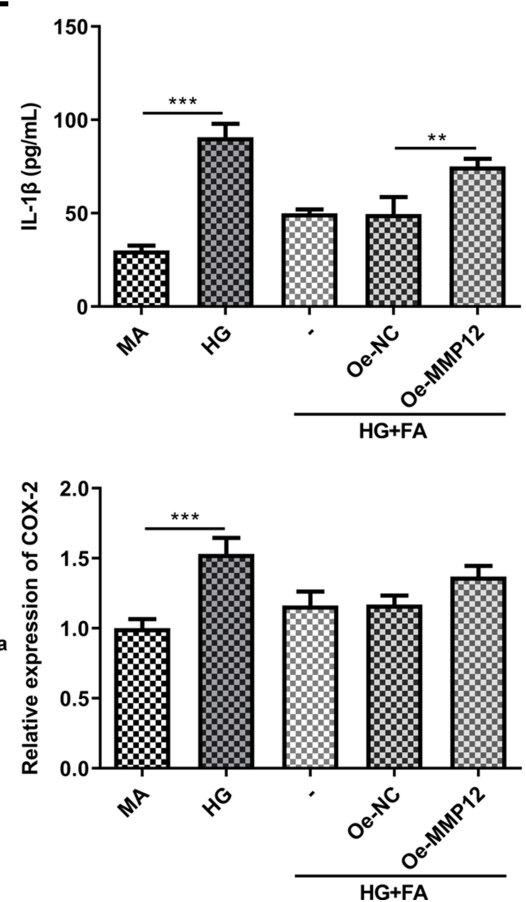

C
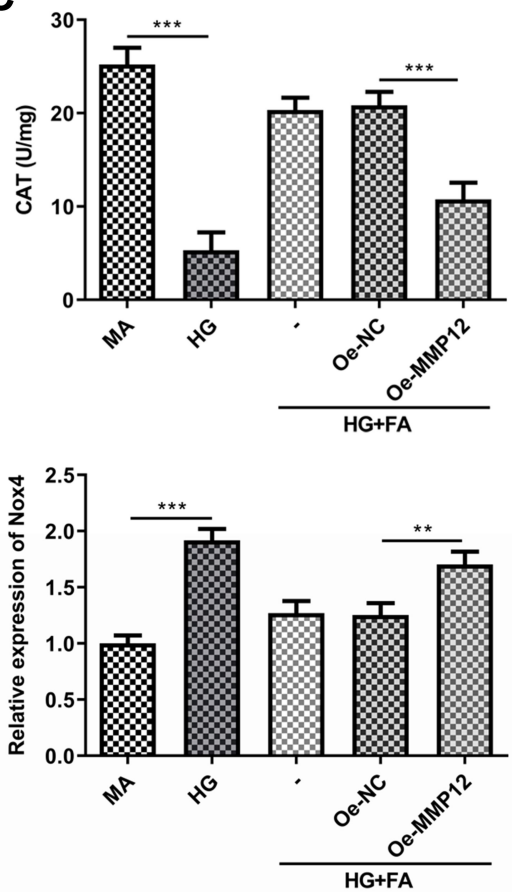

G
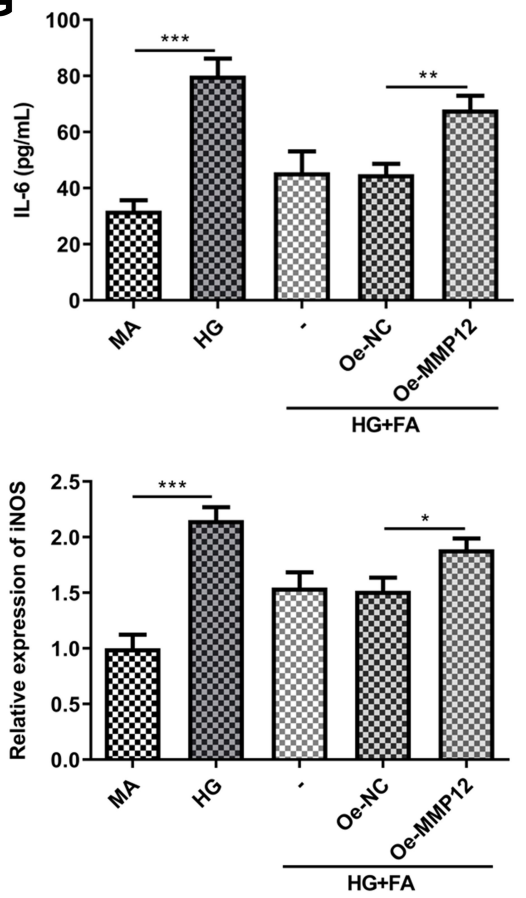

Figure 5 MMPI2 upregulation conspicuously abrogated the inhibitory effects of FA treatment on oxidative stress and inflammation in HG-induced MPC-5 cells. (A-C) The levels of MDA, SOD and CAT were examined using the corresponding commercially available kits. (D) The expression of Nox2 and Nox4 was determined with Western blotting. (E-G) The concentrations of TNF- $\alpha$, IL-I $\beta$ and IL- 6 were assessed by ELISA kits. (H) The expression of COX-2 and iNOS was measured with Western blotting. $* \mathrm{P}<0.05, * * \mathrm{P}<0.01, * * * \mathrm{P}<0.001$.

of DN, among which FA possesses a wide range of pharmacological properties in the regulation of multiple physiological activities. In the present study, we provided convincing evidence that FA protects podocytes from HG- triggered injury by inactivating MAPK signaling via MMP12 inhibition.

Accumulating study confirms that HG-induced reduction in podocyte viability and increase in apoptosis are the 

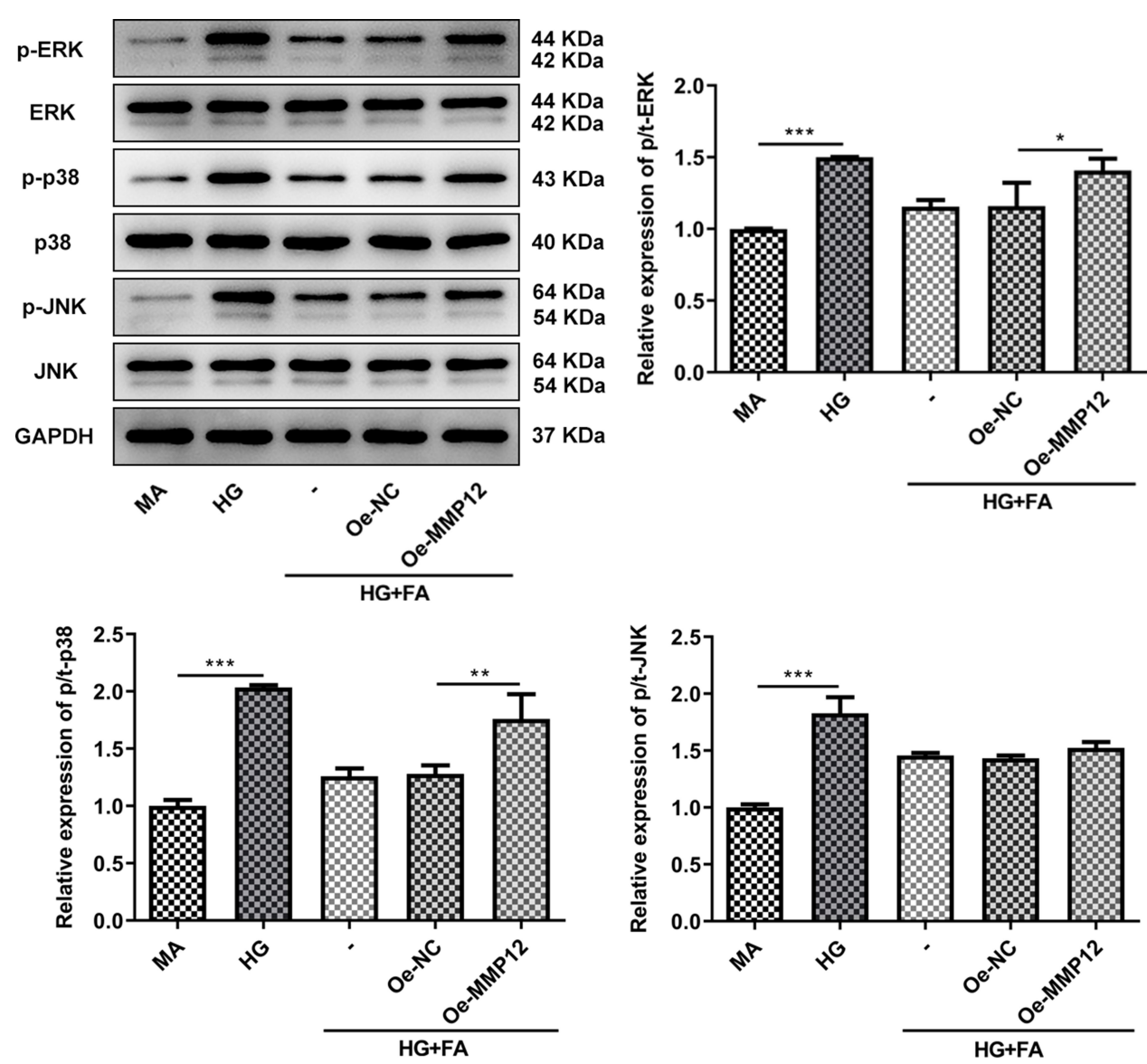

Figure 6 FA ameliorated HG-induced podocyte injury by inactivating MAPK signaling via MMPI 2 inhibition. The expression of p-ERK, P-p38 and p-JNK was determined with Western blot analysis. $* \mathrm{P}<0.05, * * \mathrm{P}<0.01$, $* * * \mathrm{P}<0.001$.

major causes of podocyte depletion, which plays a crucial role in the pathogenesis of DN. ${ }^{7,28} \mathrm{FA}$ was reported to increase the viability of lipopolysaccharide-treated RAW264.7 cells and primary lymphocytes. ${ }^{29}$ Yan et al demonstrated that FA exerts neuroprotective effects on amyloid beta $25-35$-stimulated PC12 cells by enhancing cell viability and decreasing cell apoptosis. ${ }^{30}$ In the current study, HG exposure markedly interfered with MPC-5 cell viability and intensified the percentage of apoptotic cells, which is consistent with the results of previous studies. $^{31,32}$ Importantly, FA treatment dose-dependently improved the impact of HG induction on MPC-5 cells, suggesting the protective effect of FA on podocytes under HG condition.

Additionally, oxidative stress and inflammation are reported to be closely associated with HG-induced podocyte injury in the development of DN. ${ }^{33,34}$ MDA, the end product of lipid peroxidation, is an oxidative stress marker. SOD and CAT are crucial antioxidant enzymes in the defensive system under oxidative stress condition, which together resist the podocytes to HG-induced cell death. $^{35,36}$ Nox2 and Nox4, pathologically relevant sources of ROS in HG-induced podocytes, appear to be the dominant contributors to the mediation of glomerular injury in diabetes. ${ }^{37,38}$ Additionally, the amplified and continued production of inflammatory cytokines including TNF- $\alpha$, IL- $1 \beta$ and IL- 6 can be induced by exposure of podocytes to $\mathrm{HG}^{39}{ }^{39} \mathrm{COX}-2$ and iNOS proteins are two important inflammation-related proteins during DN progression, and their induction are well documented in podocytes response to HG stimulation. ${ }^{40}$ FA, which could be a major antioxidant constituent, ${ }^{17}$ was reported to protect against lipopolysaccharide-induced inflammation in acute lung injury via up-regulating miR-124 expression. ${ }^{18}$ Moreover, it exerts anti-inflammatory effects on S. aureusstimulated primary bovine mammary epithelial cell injury through inactivation of NF-kappa $\mathrm{B}$ and MAPKs pathways. ${ }^{19}$ Apart from the previous standpoints, this study revealed that FA alleviates HG-induced oxidative stress and inflammation in podocytes. 
MMP12, a protein of the matrix metalloproteinase family, is considered to be associated with an increased risk of $\mathrm{DN}^{23} \mathrm{HG}$ exposure significantly upregulated MMP12 expression in this study implied the elevated risk of DN initiation and development, whereas FA intervention dose-dependently downregulated its expression. However, MMP12 overexpression notably reversed the protective effects of FA treatment on HG-induced MPC-5 cells. Substantial evidence exists to suggest that MAPK signaling pathway is activated when podocytes are exposed to $\mathrm{HG}$, which is further verified in the present study. ${ }^{41,42}$ Besides, MMP12 promotes the secretion of IL$1 \beta$, IL- 6 and TNF- $\alpha$ in mouse macrophages through upregulating ERK/p38 MAPK signaling pathway, and MMP12 knockdown decreases the phosphorylation levels of p38 and ERK $1 / 2 .^{43}$ In this study, significantly decreased expression levels of p-ERK, p-p38 and p-JNK in MAPK signaling in FA-treated group were observed, which was partially restored following MMP12 overexpression.

\section{Conclusion}

Taken together, results presented in the current study demonstrated that FA alleviates HG-induced oxidative stress and inflammation in podocytes. The protective effects are mediated by the inhibition of MAPK signaling via MMP12 inactivation. These findings provide experimental and scientific evidence for the potential of using FA as a therapeutic agent in the treatment of $\mathrm{DN}$.

\section{Funding}

There is no funding to report.

\section{Disclosure}

The authors declare that they have no conflicts of interest for this work.

\section{References}

1. Reutens AT. Epidemiology of diabetic kidney disease. Med Clin North Am. 2013;97(1):1-18. doi:10.1016/j.mcna.2012.10.001

2. Afkarian M, Zelnick LR, Hall YN, et al. Clinical manifestations of kidney disease among US adults with diabetes, 1988-2014. JAMA. 2016;316(6):602-610. doi:10.1001/jama.2016.10924

3. Thomas MC, Weekes AJ, Broadley OJ, Cooper ME, Mathew TH. The burden of chronic kidney disease in Australian patients with type 2 diabetes (the NEFRON study). Med J Aust. 2006;185(3):140-144. doi:10.5694/j.1326-5377.2006.tb00499.x

4. Dwyer JP, Parving HH, Hunsicker LG, Ravid M, Remuzzi G, Lewis JB. Renal dysfunction in the presence of normoalbuminuria in type 2 diabetes: results from the DEMAND Study. Cardiorenal Med. 2012;2(1):1-10. doi:10.1159/000333249
5. Abdel-Rahman EM, Saadulla L, Reeves WB, Awad AS. Therapeutic modalities in diabetic nephropathy: standard and emerging approaches. J Gen Intern Med. 2012;27(4):458-468. doi:10.1007/ s11606-011-1912-5

6. Berthier CC, Zhang H, Schin M, et al. Enhanced expression of Janus kinase-signal transducer and activator of transcription pathway members in human diabetic nephropathy. Diabetes. 2009;58(2):469-477. doi: $10.2337 / \mathrm{db} 08-1328$

7. Chen Y, Liu Q, Shan Z, et al. The protective effect and mechanism of catalpol on high glucose-induced podocyte injury. BMC Complement Altern Med. 2019;19(1):244. doi:10.1186/s12906-019-2656-8

8. Pagtalunan ME, Miller PL, Jumping-Eagle S, et al. Podocyte loss and progressive glomerular injury in type II diabetes. $J$ Clin Invest. 1997;99(2):342-348. doi:10.1172/JCI119163

9. Cai RY, Jiang JJ. LncRNA ANRIL silencing alleviates high glucose-induced inflammation, oxidative stress, and apoptosis via upregulation of MME in podocytes. Inflammation. 2020;43 (6):2147-2155. doi:10.1007/s10753-020-01282-1

10. Anil Kumar P, Welsh GI, Saleem MA, Menon RK. Molecular and cellular events mediating glomerular podocyte dysfunction and depletion in diabetes mellitus. Front Endocrinol (Lausanne). 2014;5:151. doi:10.3389/fendo.2014.00151

11. Abou-Hany HO, Atef H, Said E, Elkashef HA, Salem HA. Crocin mediated amelioration of oxidative burden and inflammatory cascade suppresses diabetic nephropathy progression in diabetic rats. Chem Biol Interact. 2018;284:90-100. doi:10.1016/j.cbi.2018.02.001

12. Xue W, Mao J, Chen Q, Ling W, Sun Y. Mogroside IIIE alleviates high glucose-induced inflammation, oxidative stress and apoptosis of podocytes by the activation of AMPK/SIRT1 signaling pathway. Diabetes Metab Syndr Obes. 2020;13:3821-3830. doi:10.2147/ DMSO.S276184

13. Qu H, Zhang Y, Wang Y, Li B, Sun W. Antioxidant and antibacterial activity of two compounds (forsythiaside and forsythin) isolated from Forsythia suspensa. J Pharm Pharmacol. 2008;60(2):261-266. doi:10.1211/jpp.60.2.0016

14. Law AHY, Yang CLH, Lau ASY, Chan GCF. Antiviral effect of forsythoside A from Forsythia suspensa (Thunb.) Vahl fruit against influenza A virus through reduction of viral M1 protein. J Ethnopharmacol. 2017;209:236-247. doi:10.1016/j.jep.2017.07.015

15. Liu CL, Su HC, Wan HY, et al. Forsythoside A exerts antipyretic effect on yeast-induced pyrexia mice via inhibiting transient receptor potential vanilloid 1 function. Int $J$ Biol Sci. 2017;13(1):65-75. doi:10.7150/ijbs. 18045

16. Wang Y, Zhao H, Lin C, Ren J, Zhang S. Forsythiaside A exhibits anti-inflammatory effects in LPS-stimulated BV2 microglia cells through activation of Nrf2/HO-1 signaling pathway. Neurochem Res. 2016;41(4):659-665. doi:10.1007/s11064-015-1731-x

17. Lu T, Piao XL, Zhang Q, Wang D, Piao XS, Kim SW. Protective effects of Forsythia suspensa extract against oxidative stress induced by diquat in rats. Food Chem Toxicol. 2010;48(2):764-770. doi:10.1016/j.fct.2009.12.018

18. Lu ZB, Yang HY, Cao HH, et al. Forsythoside A protects against lipopolysaccharide-induced acute lung injury through up-regulating microRNA-124. Clin Sci. 2020;134(19):2549-2563. doi:10.1042/ CS20200598

19. Zhang JL, Zhang Y, Huang HL, et al. Forsythoside A inhibited S. aureus stimulated inflammatory response in primary bovine mammary epithelial cells. Microb Pathog. 2018;116:158-163. doi:10.1016/j.micpath.2018.01.002

20. Lu C, Zheng SF, Liu J. Forsythiaside A alleviates renal damage in adriamycin-induced nephropathy. Front Biosci. 2020;25:526-535. doi: $10.2741 / 4818$

21. Urbschat A, Paulus P, Wiegratz I, et al. Macrophage metalloelastase-12 is detectable in human seminal plasma and represents a predictor for inflammatory processes in the male genital tract. Andrologia. 2015;47(2):153-159. doi:10.1111/and.12238 
22. Goncalves I, Bengtsson E, Colhoun HM, et al. Elevated plasma levels of MMP-12 are associated with atherosclerotic burden and symptomatic cardiovascular disease in subjects with type 2 diabetes. Arterioscler Thromb Vasc Biol. 2015;35(7):1723-1731. doi:10.1161/ ATVBAHA.115.305631

23. Tziastoudi M, Stefanidis I, Hadjigeorgiou GM, Stravodimos K, Zintzaras E. A systematic review and meta-analysis of genetic association studies for the role of inflammation and the immune system in diabetic nephropathy. Clin Kidney J. 2017;10(3):293-300. doi:10.1093/ckj/sfx008

24. Livak KJ, Schmittgen TD. Analysis of relative gene expression data using real-time quantitative PCR and the 2(-Delta Delta C(T)) method. Methods. 2001;25(4):402-408. doi:10.1006/meth.2001.1262

25. Ogurtsova K, da Rocha Fernandes JD, Huang Y, et al. IDF diabetes atlas: global estimates for the prevalence of diabetes for 2015 and 2040. Diabetes Res Clin Pract. 2017;128:40-50. doi:10.1016/j. diabres.2017.03.024

26. Zhou L, Chen X, Lu M, et al. Wnt/beta-catenin links oxidative stress to podocyte injury and proteinuria. Kidney Int. 2019;95(4):830-845. doi:10.1016/j.kint.2018.10.032

27. Asanuma K, Mundel P. The role of podocytes in glomerular pathobiology. Clin Exp Nephrol. 2003;7(4):255-259. doi:10.1007/ s10157-003-0259-6

28. Shi W, Huang Y, Zhao X, et al. Histone deacetylase 4 mediates high glucose-induced podocyte apoptosis via upregulation of calcineurin Biochem Biophys Res Commun. 2020;533(4):1061-1068. doi:10.1016/j.bbrc.2020.09.121

29. Zeng XY, Yuan W, Zhou L, Wang SX, Xie Y, Fu YJ. Forsythoside A exerts an anti-endotoxin effect by blocking the LPS/TLR4 signaling pathway and inhibiting tregs in vitro. Int $\mathrm{J} \mathrm{Mol} \mathrm{Med.} \mathrm{2017;40}$ (1):243-250. doi:10.3892/ijmm.2017.2990

30. Yan XJ, Chen TG, Zhang LW, Du HZ. Protective effects of forsythoside A on amyloid beta-induced apoptosis in PC12 cells by downregulating acetylcholinesterase. Eur $J$ Pharmacol. 2017;810:141-148. doi:10.1016/j.ejphar.2017.07.009

31. Chen Y, Yan R, Li B, et al. Silencing CCNG1 protects MPC-5 cells from high glucose-induced proliferation-inhibition and apoptosis-promotion via MDM2/p53 signaling pathway. Int Urol Nephrol. 2020;52(3):581-593. doi:10.1007/s11255-020-02383-4

32. Wang HK, Zhang YH, Xia FF, Zhang W, Chen P, Yang GA. Protective effect of silencing Statl on high glucose-induced podocytes injury via forkhead transcription factor O1-regulated the oxidative stress response. BMC Mol Cell Biol. 2019;20:15. doi:10.1186/ s12860-019-0209-0
33. Wang DZ, Jin MY, Zhao XY, et al. FGF1(Delta HBS) ameliorates chronic kidney disease via PI3K/AKT mediated suppression of oxidative stress and inflammation. Cell Death Dis. 2019;10:14.

34. Zou -H-H, Yang -P-P, Huang T-L, Zheng -X-X, Xu G-S. PLK2 plays an essential role in high D-glucose-induced apoptosis, ROS generation and inflammation in podocytes. Sci Rep. 2017;7(1):14. doi:10.1038/s41598-017-00686-8

35. Qi MY, Wang XT, Xu HL, Yang ZL, Cheng Y, Zhou B. Protective effect of ferulic acid on STZ-induced diabetic nephropathy in rats. Food Funct. 2020;11(4):3706-3718. doi:10.1039/C9FO02398D

36. Cao AL, Wang L, Chen X, et al. Ursodeoxycholic acid ameliorated diabetic nephropathy by attenuating hyperglycemia-mediated oxidative stress. Biol Pharm Bull. 2016;39(8):1300-1308. doi:10.1248/ bpb.b16-00094

37. Brandes RP, Weissmann N, Schroder K. Nox family NADPH oxidases: molecular mechanisms of activation. Free Radic Biol Med. 2014;76:208-226.

38. Nagasu H, Satoh M, Kiyokage E, et al. Activation of endothelial $\mathrm{NAD}(\mathrm{P}) \mathrm{H}$ oxidase accelerates early glomerular injury in diabetic mice. Lab Invest. 2016;96(1):25-36. doi:10.1038/labinvest.2015.128

39. Wang W, Ding XQ, Gu TT, et al. Pterostilbene and allopurinol reduce fructose-induced podocyte oxidative stress and inflammation via microRNA-377. Free Radic Biol Med. 2015;83:214-226. doi:10.1016/j.freeradbiomed.2015.02.029

40. Kim YJ, Kim YA, Yokozawa T. Pycnogenol modulates apoptosis by suppressing oxidative stress and inflammation in high glucose-treated renal tubular cells. Food Chem Toxicol. 2011;49(9):2196-2201. doi:10.1016/j.fct.2011.06.012

41. Hu RT, Li CL, Zhang X, et al. The Yi-Qi-Bu-Shen recipe attenuates high glucose-induced podocyte injury via the inhibition of IKK-I kappa B alpha-NF kappa B and ERK/P38 MAPK signaling. Int J Clin Exp Med. 2019;12(8):9782-9792.

42. Lu H, Li Y, Zhang T, et al. Salidroside reduces high-glucose-induced podocyte apoptosis and oxidative stress via upregulating Heme Oxygenase-1 (HO-1) expression. Med Sci Monit. 2017;23:10. doi:10.12659/MSM.902806

43. Guan C, Xiao Y, Li K, Wang T, Liang Y, Liao G. MMP-12 regulates proliferation of mouse macrophages via the ERK/P38 MAPK pathways during inflammation. Exp Cell Res. 2019;378(2):182-190. doi:10.1016/j.yexcr.2019.03.018

Diabetes, Metabolic Syndrome and Obesity: Targets and Therapy

Dovepress

\section{Publish your work in this journal}

Diabetes, Metabolic Syndrome and Obesity: Targets and Therapy is an international, peer-reviewed open-access journal committed to the rapid publication of the latest laboratory and clinical findings in the fields of diabetes, metabolic syndrome and obesity research. Original research, review, case reports, hypothesis formation, expert opinion and commentaries are all considered for publication. The manuscript management system is completely online and includes a very quick and fair peer-review system, which is all easy to use. Visit http://www.dovepress.com/testimonials.php to read real quotes from published authors. 